\title{
Persistência de Distúrbios Perfusionais Miocárdicos após Intervenção Coronária Percutânea com Êxito: Dependência de Fatores Microcirculatórios
}

\author{
J. Antonio Marin-Neto', Moysés de O. Lima Filho', Marcus V. Simões"1, \\ A. O. Pintya ${ }^{1}$, Pedro E. Horta ${ }^{2}$, Siguemituzo Ariê $\hat{e}^{2}$ (in memoriam)
}

\begin{abstract}
RESUMO
Introdução: A persistência transitória de defeitos perfusionais imediatamente após intervenção coronária percutânea bem sucedida para correção de estenoses coronárias é bem documentada. Método: Para testar a hipótese de que tais anormalidades perfusionais sejam associadas a distúrbios microcirculatórios causados por microembolização coronária, comparou-se a intensidade e extensão desses defeitos perfusionais detectados com cintilografia miocárdica em grupos randomicamente constituídos de pacientes tratados com angioplastia coronária por balão $(\mathrm{AB})$ ou submetidos a aterectomia rotacional complementada por balão $(A R+B)$. As características clínicas e angiográficas foram comparáveis nos dois grupos, assim como o sucesso do procedimento de angioplastia coronária. Resultados: Antes da intervenção coronária percutânea, o índice de defeito miocárdico, englobando a extensão e a gravidade da hipoperfusão, foi comparável nos dois grupos, na condição de estresse $(A B=7,72 \pm 1,91$ vs $A R+B=8,61 \pm$ $3,38)$ e de repouso ( $A B=3,11 \pm 1,22$ vs $A R+B=2,40 \pm 1,63)$. Após o procedimento, o índice de defeito perfusional decresceu em ambos os grupos durante o estresse, mas com significância estatística apenas no grupo $A B=3,96 \pm 1,40$ vs $A R+B$ $=3,71 \pm 1,89$. O contraste entre os dois grupos se acentuou na condição de repouso após a intervenção coronária: o índice de defeito decresceu de forma marginalmente significante no grupo $A B$ para 1,46 $\pm 0,66$ e aumentou, embora sem

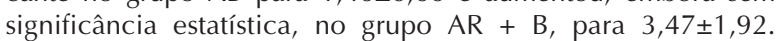
Conclusão: Esses resultados são compatíveis com o conceito de que a persistência transitória de defeitos perfusionais após angioplastia coronária bem sucedida seja dependente de distúrbios microcirculatórios associados à microembolização durante o procedimento.
\end{abstract}

DESCRITORES: Microcirculação. Aterectomia coronária. Angioplastia transluminal percutânea coronária.

\footnotetext{
1 Unidade de Cardiologia Intervencionista, Divisão de Cardiologia, Departamento de Clínica Médica, Hospital das Clínicas, Faculdade de Medicina de Ribeirão Preto da Universidade de São Paulo, Ribeirão Preto, SP.

${ }^{2}$ Instituto do Coração, Faculdade de Medicina da Universidade de São Paulo, São Paulo, SP.

Correspondência: J. Antonio Marin-Neto. Unidade de Cardiologia Intervencionista, Divisão de Cardiologia, Departamento de Clínica Médica, Hospital das Clínicas, Faculdade de Medicina de Ribeirão Preto, USP. Avenida Bandeirantes, 3900 - Campus da USP em Ribeirão Preto - SP - CEP 14048-900.

Tel.: (16) 3633-4010 • E-mail: marin_neto@yahoo.com

Recebido em: 17/01/2007 • Aceito em: 21/03/2007
}

\section{SUMMARY}

\author{
Myocardial Perfusion Disturbances Persisting after \\ Successful Percutaneous Coronary Angioplasty: \\ Relationship to Microcirculatory Derangements
}

Introduction: The transitory persistence of perfusion defects immediately after successful percutaneous coronary interventions to correct coronary stenosis is well known. Methods: To test the hypothesis that such perfusion abnormalities are associated with microcirculatory disorders caused by coronary microembolization we compared the intensity and extent of these perfusion defects detected using myocardial scintigraphy in groups of patients randomly assigned to coronary balloon angioplasty (BA) or to rotational atherectomy plus balloon angioplasty $(R A+B)$. The clinical and angiography characteristics were comparable in both groups, as well as the success of the coronary angioplasty procedure. Results: Before the percutaneous coronary intervention the myocardium defect index, related to the extent and severity of hypoperfusion, was comparable for the two groups, both under stress $(\mathrm{AB}=$ $7.72 \pm 1.91$ vs. $R A+B=8.61 \pm 3.38)$ and at rest $(A B=3.11 \pm 1.22$ vs. $R A+B=2.40 \pm 1.63)$. After the procedure, the perfusion defect index decreased for both groups during stress, but with statistical significance only in the $A B$ Group $=3.96 \pm 1.40$ vs $R A+B=3.71 \pm 1.89$. The difference between the two groups was greater at rest after the coronary intervention procedure: the defect index decreased with marginal significance for the $\mathrm{AB}$ Group to $1.46 \pm 0.66$ and increased, though without statistical significance, for the RA + B Group to $3.47 \pm 1.92$. Conclusion: These results are compatible with the notion that transitory persistence of perfusion defects after successful coronary angioplasty are dependent on microcirculatory disorders associated to microembolization during the procedure.

DESCRIPTORS: Microcirculation. Atherectomy, coronary. Angioplasty, transluminal, percutaneous coronary. 
Marin-Neto JA, et al. Persistência de Distúrbios Perfusionais Miocárdicos após Intervenção Coronária Percutânea com Êxito: Dependência de Fatores Microcirculatórios. Rev Bras Cardiol Invas 2007; 15(2): 107-114.

A cintilografia miocárdica de perfusão (CMP) desde muito cedo foi empregada para embasar a indicação da intervenção coronária percutânea (ICP) para tratamento de lesões coronárias obstrutivas, e para verificar o sucesso do procedimento. O método mostrou-se efetivo sob condições clínicas diversas, desde intervenções em pacientes estáveis tratados eletivamente $^{1}$, passando por aqueles tratados em virtude de recorrência de angina após revascularização cirúrgi$\mathrm{ca}^{2}$ e culminando na avaliação dos resultados da angioplastia primária para recanalização coronária durante infarto agudo do miocárdio (IAM) ${ }^{3}$. Entretanto, a paradoxal persistência de defeitos perfusionais durante os primeiros dias ou semanas que se seguem à revascularização miocárdica bem sucedida foi documentada desde a década de $1980^{4}$, e confirmada por estudos posteriores $^{5,6}$. Em conseqüência disso, é conduta empírica bastante difundida e aceita clinicamente, que se aguarde algumas semanas após a ICP, antes de se proceder a verificação do sucesso do procedimento, com o método da $\mathrm{CMP}^{7}$.

Tais defeitos perfusionais, ocorrrendo em áreas miocárdicas supridas por artérias coronárias subepicárdicas que não mais apresentam estenoses hemodinamicamente significativas, costumam se associar a recorrência de precordialgia, na maioria das vezes, atípica para isquemia cardíaca $^{8}$. Embora esses defeitos perfusionais persistentes após ICP bem sucedida possam ser do tipo reversível (sugerindo isquemia indutível apenas durante estresse), também têm sido descritos na condição de repouso, indiciando a possibilidade de isquemia miocárdica incessante $^{1-8}$. Os defeitos perfusionais têm sido relatados em estudos cintilográficos empregando marcadores de fluxo e viabilidade miocárdica, como o Tálio-201 1,2,4-6,8,9, ou traçadores predominantemente indicadores da distribuição regional do fluxo coronário, como o $\mathrm{Tc}^{99 \mathrm{~m}}$ sestamibi ${ }^{3}$. Além disso, essas alterações persistentes da distribuição regional de fluxo miocárdico também são encontradas quando outros métodos são empregados, como a resolução das elevações de segmento $\mathrm{ST}^{10}$ e a ecocardiografia de contraste ${ }^{11}$.

O intrigante fenômeno da persistência de defeitos perfusionais após ICP bem sucedida tem sido observado em variados contextos clínicos, desde condições eletivas em pacientes estáveis até a situação de IAM tratado com angioplastia primária. No início, quando a persistência de distúrbios perfusionais era verificada após ICP eletiva de pacientes estáveis, aventou-se a possibilidade de que vários fatores diretamente ligados à ICP pudessem contribuir para explicá-los: manutenção de lesão residual $>50 \%$, acometimento de ramos secundários ao vaso dilatado e presença de linhas de dissecção restringindo o fluxo coronário ${ }^{5}$. Entretanto, a compreensão fisiopatológica da gênese desses distúrbios perfusionais ainda é muito obscura, pois podem ocorrer após ICP absolutamente bem sucedida, isto é, na ausência de quaisquer fatores identificáveis ao nível da circulação subepicárdica para a área miocárdica que os apresenta. Nesse sentido, hipótese alternativa é de que esses defeitos perfusionais sejam causados por anormalidades microcirculatórias coronárias.

Se a persistência de defeitos miocárdicos perfusionais após ICP bem sucedida for causada por distúrbios microcirculatórios, dois seriam os principais mecanismos responsáveis: anomalias de regulação do fluxo e calibre coronários ao nível microvascular, e microembolização periférica. Em pacientes submetidos a ICP para tratamento de lesões instáveis, em que há elevada tendência pró-trombótica, a embolia em nível microcirculatório torna-se bastante provável. Entretanto, não há relato na literatura de investigações comparando a incidência do fenômeno em grupos de pacientes tratados por ICP na vigência e ausência de quadros clínicos instáveis.

Também restaria explicar a ocorrência do fenômeno quando ocorre em pacientes estáveis, em que se realiza ICP eletiva, sem carga ou tendência trombótica apreciável. Neste contexto, o método da aterectomia rotacional foi implicado na gênese de distúrbios microcirculatórios, possivelmente causados por microembolização coronária periférica. Nesta investigação, os defeitos perfusionais foram detectados durante o procedimento, mas desapareceram poucos dias após ${ }^{12}$. Entretanto, não foram, até o presente, conduzidos estudos comparando a incidência e gravidade de defeitos perfusionais persistindo transitoriamente após ICP bem sucedida, em grupos de pacientes estáveis, submetidos a técnicas de angioplastia com mais ou menos probabilidade de desencadear microembolização coronária. Este foi o intuito da presente investigação, explorandose o modelo da aterectomia rotacional, comparativamente à ICP sem esse método ablativo de ateroma, para testar a hipótese de que distúrbios microcirculatórios causando defeitos cintilográficos persistentes transitoriamente dependam de microembolização coronária.

\section{MÉTODO}

\section{População estudada}

Entre julho de 1995 a outubro de 1996, desenvolveu-se esta investigação prospectiva para comparação dos efeitos da aterectomia rotacional complementada por balão e da angioplastia por balão isoladamente sobre distúrbios perfusionais miocárdicos avaliados cintilograficamente. Nesse período, 36 pacientes, com idade superior a 18 anos, de ambos os gêneros, sem limites de idade, em pleno exercício de suas faculdades mentais e com capacidade de prestar informações e entender o espírito da pesquisa, portadores de 38 lesões coronarianas obstrutivas e com evidência clínica cintilográfica de isquemia miocárdica foram selecionados para participar do estudo. Todos deram consentimento escrito, tendo o projeto sido aprovado pelas Comissões de Ética em Pesquisa de ambas as instituições em que os estudos foram conduzidos, a saber, o Hospital 
Marin-Neto JA, et al. Persistência de Distúrbios Perfusionais Miocárdicos após Intervenção Coronária Percutânea com Êxito: Dependência de Fatores Microcirculatórios. Rev Bras Cardiol Invas 2007; 15(2): 107-114.

das Clínicas da Faculdade de Medicina de Ribeirão Preto, USP (22 pacientes), e o Instituto do Coração da Faculdade de Medicina da Universidade de São Paulo (14 pacientes).

Além dos critérios de inclusão já mencionados, todos os pacientes apresentavam critérios angiográficos adicionais para serem arrolados no estudo: lesões reduzindo, pela estimativa visual, o D.L.R. (diâmetro luminal de referência) em $\geq 70 \%$. Ademais, esses pacientes eram portadores de lesões com possível indicação de aterectomia rotacional: calcificadas; excêntricas; reestenóticas; ostiais; lesões com insucesso prévio de angioplastia por balão, com apenas o guia metálico sendo capaz de ultrapassá-las.

Na fase de seleção dos pacientes, os critérios de exclusão aplicados foram: período gestacional; presença de trombo visível angiograficamente; lesões em tronco de artéria coronária esquerda não protegido por cirurgia de revascularização miocárdica prévia; lesões em pontes de veia safena degeneradas; dissecções extensas em artérias tratadas por angioplastia coronariana no período de um mês; lesões maiores que $20 \mathrm{~mm}$ de comprimento; lesões em vasos com diâmetro luminal de referência $<2,0 \mathrm{~mm}$.

Preenchidos os critérios de inclusão e afastados os de exclusão, os 36 pacientes, estáveis clinicamente e portando 38 lesões coronárias julgadas passíveis de abordagem por aterectomia rotacional, foram considerados para ICP eletiva, e arrolamento no estudo. Em seguida, foram randomicamente alocados para tratamento por angioplastia por balão (18 pts - 20 lesões) ou aterectomia rotacional complementada com angioplastia por balão (18 pts - 18 lesões).

\section{Intervenção coronária percutânea}

A abordagem vascular foi femoral em todos os pacientes, exceto em dois, nos quais extrema tortuosidade ilíaca forçou o acesso braquial. Nestes dois casos, randomizados para aterectomia rotacional, o cateter-guia tipo Stertzer $8 \mathrm{~F}$ usado impedia a passagem da oliva do aterótomo, possibilitando apenas o uso de cateter-balão. Também em outros dois pacientes, a aterectomia rotacional programada pela aleatorização foi substituída por angioplastia com balão apenas, por não se conseguir a ultrapassagem do fio-guia 0,009", sobre o qual desliza o aterótomo, através da lesão. Em ambos os casos, houve sucesso na ultrapassagem do fio-guia 0,014" convencional. No grupo randomizado para angioplastia por balão, não houve mudança de procedimento, para a aterectomia rotacional.

Foram analisadas as coronariografias realizadas imediatamente antes da passagem do fio-guia, e 15 minutos após a última dilatação por balão (com ou sem uso prévio do aterótomo). A análise angiográfica coronariana quantitativa foi sempre conduzida "offline", por método previamente validado: C.A.A.S. II
(Coronary Angiography Analysis System) ${ }^{13}$, na projeção em que se que obtinha a melhor imagem da lesão e do vaso tratado.

Todos os pacientes estavam em uso de ácido acetilsalicílico (AAS) 200 mg, durante a ICP. Ficou a critério do operador administrar mononitrato ou nitroglicerina intracoronária durante o procedimento, o que geralmente foi feito diante de espasmo do vaso tratado. Os pacientes receberam 15000 U.I. de heparina intravenosa, imediatamente após a abordagem vascular, e doses adicionais de 5000 U.I, a cada hora de duração do procedimento.

Foi utilizada apenas uma oliva em cada uma das lesões tratadas com aterectomia rotacional, e o critério utilizado para escolha de seu diâmetro foi o de não ultrapassar a relação de 0,7 com o valor do diâmetro de referência do vaso tratado, por estimativa visual do operador. Este valor foi definido protocolarmente em virtude de estudos prévios terem demonstrado maior risco de complicações - perfurações, dissecções, oclusões agudas - quando valores de relação acima de 0,7 a 0,8 eram ultrapassados ${ }^{14,15}$.

O número de passagens do aterótomo por cada lesão não foi definido protocolarmente, sendo deixado a critério dos operadores. A escolha do balão também baseou-se em estimativa visual, procurando-se não ultrapassar relação de 1:1 entre o calibre do vaso tratado e o valor nominal do diâmetro do balão a ser utilizado.

Após a última passagem do aterótomo, nova coronariografia foi realizada e, em seqüência, trocava-se o aterótomo pelo cateter-balão para a complementação do procedimento com a dilatação coronária. O número de dilatações também ficou a critério dos operadores, não tendo sido imposto nenhum tipo de restrição protocolar quanto a este item, assim como quanto à eventual troca de cateter-balão.

A definição de sucesso do tratamento proposto foi redução da obstrução para $<50 \%$ do diâmetro luminal de referência, ao término do procedimento, na ausência de complicações graves (infarto agudo do miocárdio, cirurgia de revascularização ou óbito).

Terminado o procedimento intervencionista, os pacientes permaneciam internados por 48 horas, sendo medicados com bloqueadores de canais de cálcio (Diltiazem 30 a 60 mg via oral, a cada 8 horas), AAS (200 mg via oral, diariamente) e nitratos (dinitrato ou mononitrato ambos $10 \mathrm{mg}$ via oral, a cada 8 horas). Os vasodilatadores continuaram durante o primeiro mês, e o AAS, indefinidamente. $\mathrm{O}$ uso de heparina foi restrito, durante as primeiras 24 horas, aos pacientes com presença de linhas de dissecção.

Em período médio de 7 dias (2 a 21) após a ICP, os pacientes foram submetidos a novo estudo de cintilografia de perfusão miocárdica, com os mesmos métodos do estudo nuclear anterior ao procedimento. 
Marin-Neto JA, et al. Persistência de Distúrbios Perfusionais Miocárdicos após Intervenção Coronária Percutânea com Êxito: Dependência de Fatores Microcirculatórios. Rev Bras Cardiol Invas 2007; 15(2): 107-114.

\section{Cintilografia miocárdica de perfusão}

O exame cintilográfico de perfusão miocárdica com ${ }^{201}$ Tálio ou ${ }^{99 m}$ sestamibi, em condições de estresse físico ou farmacológico com dipiridamol, foi sempre conduzido pela técnica de tomografia computadorizada de emissão de fótons singulares (SPECT). Os estudos foram realizados em gâma-câmara Siemens modelo Orbiter, por captação das imagens em 32 cortes tomográficos, em angulação global de $180^{\circ}$, iniciando-se em oblíqua anterior direita a $40^{\circ}$.

A análise qualitativa foi realizada a partir de reconstrução das imagens tomográficas em projeções ortogonais clássicas, de eixo menor e eixos longos longitudinal e horizontal, e sempre realizada por dois experientes investigadores, mascarados com relação ao período pré ou pós-procedimento, e também com relação ao grupo de alocação randomizada.

A análise quantitativa dos defeitos de perfusão, nas regiões supridas pelas artérias tratadas, foi efetuada utilizando-se mapas polares com reconstrução de cortes radiais. A redução na captação do "pixel" foi considerada significante quando excedeu 2,5 desviospadrões da população normal ${ }^{16}$. Foi calculado um índice de defeito, englobando a extensão do mesmo relativamente à área de projeção da superfície ventricular esquerda nos mapas polares, multiplicada pela média da gravidade da hipocaptação dos "pixels".

\section{Análise estatística}

$\mathrm{Na}$ avaliação dos resultados comparativos dos grupos, foi considerado o tratamento final destinado a cada paciente e não o tratamento para o qual o paciente foi randomizado. Este desvio do princípio da intenção de tratamento, aplicável usualmente a análises de grandes grupos randomizados, se justifica pelo reduzido número de pacientes em cada grupo, neste estudo.

As variáveis quantitativas e semi-quantitativas foram analisadas utilizando-se os testes de Wilcoxon e de Friedman. Os testes de qui quadrado ou exato de Fischer foram utilizados para comparação das variáves qualitativas nos dois grupos. O nível crítico de significância estatístico foi estabelecido como 0.05.

\section{RESULTADOS}

\section{Características clínicas dos grupos}

Como exposto na Tabela 1, ambos os grupos apresentavam a mesma média de idade, 57,7 anos, variando de 43 a 75 anos, no grupo tratado por aterectomia rotacional complementada por balão ( $A R+A B)$, e de 37 a 77 anos, no grupo tratado por angioplastia por balão isoladamente (AB). Também apresentavam o mesmo número de pacientes do sexo masculino, 13, correspondendo a $81,2 \%$ do grupo $A R+A B$ e $65 \%$ do grupo $A B$. Observou-se predominância de pacientes com angina instável no grupo $A R+A B(43,7 \%)$ e de angina estável no grupo $\mathrm{AB}(55 \%)$ e ambos os grupos apresentavam proporção comparável de pacientes com IAM prévio. Também foram superponíveis as proporções de indivíduos com diabete melito, dislipidemia, tabagismo, hipertensão arterial sistêmica e de história familiar para coronariopatia precoce, em ambos os grupos. Antecedente de ICP com reestenose era presente em um paciente de cada grupo, e de revascularização cirúrgica em apenas um outro caso, do grupo $A R+B$.

\section{Características angiográficas dos grupos}

A Tabela 2 mostra que, dentre as características morfológicas basais avaliadas, excentricidade, irregularidades de contorno, localização ostial ou em bifurcação e presença de calcificação, a única característica que mostrou diferença significante de proporção entre os dois grupos foi a localização da lesão em bifurcação, presente em $36,3 \%$ das lesões tratadas por angioplastia por balão isoladamente, vs $6,2 \%$ dos casos, no grupo tratado por aterectomia rotacional complementada por balão $(p=0,034)$. Em resumo, no grupo $A R+A B$, aproximadamente $70 \%$ das lesões foram consideradas complexas (tipo B2 ou C). No grupo AB, 45,4\% das lesões eram B1 e 54,6\%, B2, não havendo lesões tipo C neste grupo. Nenhum paciente com lesão tipo A foi incluído neste estudo, apesar de isto não constituir critério de exclusão.

A média do diâmetro luminal de referência de ambos os grupos foi comparável: 2,61 mm ( $A R+A B)$ e $2,48 \mathrm{~mm}(\mathrm{AB})$. Em relação às outras características

TABELA 1

Características clínicas dos pacientes submetidos a aterectomia rotacional complementada por balão (AR + AB) ou a angioplastia por balão isoladamente (AB)

\begin{tabular}{lrrrrr}
\hline & \multicolumn{2}{c}{$\mathbf{A R}+\mathbf{A B}$} & & \multicolumn{2}{c}{$\mathbf{A B}$} \\
\cline { 2 - 3 } \cline { 6 - 7 } & $\mathbf{N}$ & $\mathbf{\%}$ & & $\mathbf{N}$ & $\mathbf{\%}$ \\
\hline Assintomáticos & 3 & 18,7 & & 2 & 10,0 \\
Angina estável & 6 & 37,5 & & 11 & 55,0 \\
Angina instável & 7 & 43,7 & & 7 & 35,0 \\
IAM prévio & 3 & 18,7 & & 4 & 20,0 \\
ACTC prévia & 1 & 6,2 & & 2 & 10,0 \\
Reestenose & 1 & 6,2 & & 1 & 5,0 \\
Rev. Mioc. Prévia & 1 & 6,2 & & -16 & -10 \\
Hip. Arterial & 11 & 68,7 & & 16 & 80,0 \\
Diab. Melitus & 3 & 18,7 & & 3 & 15,0 \\
Dislipidemia & 7 & 43,7 & & 9 & 45,0 \\
Hist. Familiar & 6 & 37,5 & & 11 & 55,0 \\
Tabagismo & 9 & 56,2 & & 12 & 60,0 \\
Total de pac. & 16 & 100,0 & & 20 & 100,0 \\
& & & &
\end{tabular}

p=NS; Rev. Mioc.= Revascularização Miocárdica; Hip.= Hipertensão; Diab= Diabete; Hist= História; Pac= Pacientes. 
Marin-Neto JA, et al. Persistência de Distúrbios Perfusionais Miocárdicos após Intervenção Coronária Percutânea com Êxito: Dependência de Fatores Microcirculatórios. Rev Bras Cardiol Invas 2007; 15(2): 107-114.

angiográficas quantitativas analisadas, também não houve diferença estatisticamente significante entre os dois grupos, sendo a média do diâmetro luminal mínimo basal igual a 0,83 e 0,71 mm, e da porcentagem de estenose de 68,2 e 71,3\% para $A R+A B$ e $A B$, respectivamente (Tabela 2 ).

\section{Resultados da ICP}

O índice de sucesso clínico e angiográfico dos procedimentos foi de 93,2\%, para o grupo tratado por aterectomia rotacional complementada por balão (RA

TABELA 2

Características angiográficas das lesões tratadas

\begin{tabular}{|c|c|c|c|c|}
\hline & \multicolumn{2}{|c|}{$\mathrm{AR}+\mathrm{AB}$} & \multicolumn{2}{|c|}{$\mathbf{A B}$} \\
\hline & $\mathbf{N}$ & $\%$ & $\mathbf{N}$ & $\%$ \\
\hline Excentricidade & 13 & 81,2 & 14 & 63,6 \\
\hline Irreg. de cont. & 3 & 18,8 & 3 & 13,6 \\
\hline Bifurcação* & 1 & 6,2 & 8 & 36,3 \\
\hline Local ostial & 3 & 18,8 & 2 & 9,0 \\
\hline Calcif. Disc & 3 & 18,8 & 3 & 13,6 \\
\hline Calcif. Mod & 2 & 12,5 & 2 & 9,0 \\
\hline Tipo B1 & 5 & 31,2 & 10 & 45,4 \\
\hline Tipo B2 & 8 & 50,0 & 12 & 54,6 \\
\hline Tipo C & 3 & 18,8 & - & - \\
\hline DA & 8 & 50,0 & 10 & 45,4 \\
\hline CD & 7 & 43,7 & 4 & 18,2 \\
\hline CX & - & - & 3 & 13,6 \\
\hline Diagonal & - & - & 3 & 13,6 \\
\hline Marginal Esq & 1 & 63,7 & 2 & 9,1 \\
\hline Total de lesões & 16 & 100,0 & 22 & 100,0 \\
\hline Comprimento (mm) & 17, & $\pm 8,9$ & 13 & $\pm 4,8$ \\
\hline $\mathrm{DLR}(\mathrm{mm})$ & 2,61 & $\pm 0,40$ & 2,48 & $\pm 0,35$ \\
\hline $\mathrm{DLM}(\mathrm{mm})$ & 0,83 & $\pm 0,21$ & 0,70 & $\pm 0,30$ \\
\hline \% Estenose & 68, & $\pm 5,8$ & 71,3 & $\pm 10,1$ \\
\hline
\end{tabular}

${ }^{*} p=0,034$ - Abreviações: Irreg.= irregularidade; cont. = contorno; calcif.= calcificação; $\mathrm{DA}=$ descendente anterior; $\mathrm{CD}=$ coronária direita; $C X=$ circunflexa; $E s q=$ esquerdo; $D L R=$ diâmetro luminal de referência; $\mathrm{DLM}=$ diâmetro luminal mínimo.
+ BA) e de 95\%, para o grupo tratado por angioplastia por balão isoladamente (BA). Não houve significância estatística para esta pequena diferença no índice de sucesso. Não foi evidenciado o fenômeno de "no reflow"em qualquer paciente de ambos os grupos.

Os parâmetros angiográficos de relação entre os dispositivos utilizados e a quantificação vascular evidenciaram o mesmo valor para a relação balão-artéria entre os dois grupos, que foi em torno de 1,0. A relação aterótomo-artéria foi, em média, 0,68, com um desvio-padrão de 0,10 .

Apesar de o diâmetro luminal mínimo antes do procedimento ter sido comparável entre os dois grupos, esta variável apresentou incremento significativamente maior, 15 minutos após a última dilatação, no grupo tratado pela técnica átero-ablativa complementada por balão. Apesar disso, a porcentagem de estenose calculada 15 minutos após a última dilatação não evidenciou diferença significante estatisticamente entre os grupos (Tabela 3).

\section{Complicações}

Um paciente do grupo tratado por aterectomia rotacional apresentou oclusão aguda de artéria coronária descendente anterior, aproximadamente 30 minutos após o término do procedimento. Este paciente teve três paradas cardiorrespiratórias revertidas, antes e durante novo procedimento subseqüente, durante o qual implantou-se "stent" de Palmaz-Schatz para recanalização arterial e estabilização do quadro hemodinâmico.

Em outro paciente, tratado com angioplastia por balão em coronária direita, ocorreu dissecção espiralada tipo E e fluxo TIMI-2. Entretanto, evoluiu assintomaticamente, sem alteração eletrocardiográfica e com discreta elevação enzimática, sendo que, após uma semana do procedimento, nova cineangiocoronariografia evidenciou patência da artéria coronária direita com fluxo TIMI-3 e lesão residual de apenas 23\% no local tratado.

\section{Resultados cintilográficos perfusionais}

Os valores de duplo produto [freqüência cardíaca (bpm) x pressão arterial sistólica $(\mathrm{mmHg})]$, no momento da injeção do marcador radioativo, foram compa-

TABELA 3

Variação dos diâmetros luminais mínimos (em $\mathrm{mm})$ e \% de estenose antes da primeira manobra de ICP, e 15 minutos após a última dilatação com balão

\begin{tabular}{|c|c|c|c|c|}
\hline & \multicolumn{2}{|c|}{ DLM } & \multicolumn{2}{|c|}{$\%$ Estenose } \\
\hline & Pré & $15 \mathrm{~min}$ & Pré & $15 \mathrm{~min}$ \\
\hline $\mathrm{AB}$ & $0,72 \pm 0,30$ & $1,65 \pm 0,37^{*}$ & $71,4 \pm 10,1$ & $34,1 \pm 14,9$ \\
\hline
\end{tabular}


Marin-Neto JA, et al. Persistência de Distúrbios Perfusionais Miocárdicos após Intervenção Coronária Percutânea com Êxito: Dependência de Fatores Microcirculatórios. Rev Bras Cardiol Invas 2007; 15(2): 107-114.

ráveis entre os dois grupos, bem como se mantiveram superponíveis em ambos, quando foram comparados aos respectivos níveis antes e após a ICP (Tabela 4).

No grupo tratado por $A R+A B$, o índice de defeito (ID) na condição de estresse diminuiu de 8,61 $\pm 3,38$, na condição pré-procedimento, para 3,71 $\pm 1,89$, após o procedimento, diferença não significante estatisticamente $(p=0,08)$. Neste mesmo grupo, o ID em repouso aumentou (também sem significância estatística) de $2,40 \pm 1,63$, na condição pré-procedimento, para 3,47 $\pm 1,92$, após o procedimento - Tabela 5 .

Em contraste, no grupo tratado por $A B$, o ID em estresse reduziu-se de $7,72 \pm 1,91$, antes do procedimento, para $3,96 \pm 1,40$, após o procedimento $(p=0,03)$. Neste grupo, também se observou diminuição do ID em repouso, passando de 3,11 $\pm 1,22$, antes do procedimento, para 1,46 $\pm 0,66$, após a ICP, com valor limítrofe de $p=0,06$ (Tabela 5). Um exemplo ilustrativo de defeito perfusional persistente durante estudo cintilográfico conduzido precocemente após ICP, e evanescente depois de seguimento tardio, aparece na Figura 1.

\section{DISCUSSÃO}

Os presentes resultados corroboram observações anteriores, de persistência transitória de distúrbios perfusionais precocemente após ICP bem sucedida em pacientes com lesões coronárias estáveis. Assim, um desses estudos descreveu a persistência de isquemia miocárdica, em 12 de 43 pacientes submetidos a ICP

TABELA 4

Duplo produto (FC X PAS) durante estresse induzido para o estudo cintilográfico de perfusão miocárdica

\begin{tabular}{lcc}
\hline & Pré & Pós \\
\hline$A R+A B$ & $24237 \pm 4681$ & $25808 \pm 7320$ \\
$A B$ & $26207 \pm 6300$ & $26333 \pm 6570$ \\
\hline$p=N S$ & & \\
\hline
\end{tabular}

com sucesso, nas primeiras duas semanas após o procedimento, e subseqüente e total reversão da isquemia apenas por volta do terceiro mês ${ }^{4}$. Em outra observação, registrou-se melhora precoce da perfusão miocárdica em apenas $61 \%$ dos pacientes submetidos a ICP bem sucedida ao nível subepicárdico ${ }^{6}$. Outro estudo, realizado com 25 pacientes tratados com ICP também com resultado adequado, relatou em 11 deles persistência da hipoperfusão miocárdica pelo estudo cintilográfico realizado na primeira semana após o procedimento e reversão completa do fenômeno após três meses ${ }^{9}$. Tais anormalidades perfusionais foram também descritas em 30\% dos pacientes de outra investigação, com CMP realizada até 6 semanas após o procedimento terapêutico ${ }^{8}$.

Em adição a essas informações da literatura, nesta investigação, enquanto após ICP apenas com balão a intensidade e extensão do defeito perfusional na região

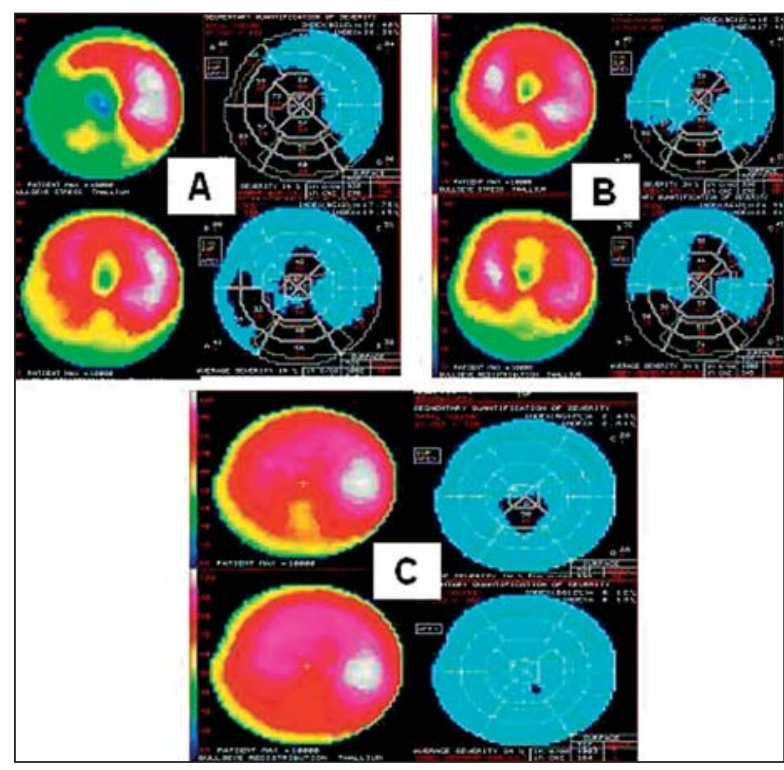

Figura 1 - Mapas polares de imagens cintilográficas obtidas antes do procedimento (A), e 5 dias (B) e 4 meses após ICP (C). Em cada painel, as imagens superiores são da fase de estresse e as inferiores da fase de repouso.

TABELA 5

Variação do Índice de Defeito (ID) em Estresse (STR) e Repouso (REP) pela cintilografia de perfusão miocárdica antes e após o procedimento terapêutico

\begin{tabular}{|c|c|c|c|c|}
\hline & \multicolumn{2}{|c|}{ Pré } & \multicolumn{2}{|c|}{ Pós } \\
\hline & ID - STR & ID - REP & ID - STR & ID - REP \\
\hline $\mathrm{AR}+\mathrm{AB}$ & $8,61 \pm 3,38$ & $2,40 \pm 1,63$ & $3,71 \pm 1,89 \bullet$ & $3,47 \pm 1,92$ \\
\hline $\mathrm{AB}$ & $7,72 \pm 1,91$ & $3,11 \pm 1,22$ & $3,96 \pm 1,40^{*}$ & $1,46 \pm 0,66^{\#}$ \\
\hline
\end{tabular}


Marin-Neto JA, et al. Persistência de Distúrbios Perfusionais Miocárdicos após Intervenção Coronária Percutânea com Êxito: Dependência de Fatores Microcirculatórios. Rev Bras Cardiol Invas 2007; 15(2): 107-114.

suprida pela artéria tratada reduziram-se significativamente tanto em estresse como em repouso, quando a ICP envolveu a aterectomia rotacional, ocorreu menor diminuição do defeito perfusional, sob estresse, verificando-se, inclusive, aumento do mesmo na condição de repouso. Tais diferenças ocorreram a despeito de os resultados dos procedimentos terem sido inteiramente comparáveis nos dois grupos, quanto ao alívio da estenose na artéria subepicárdica responsável pela área miocárdica apresentando persistência dos defeitos perfusionais.

Os resultados deste estudo podem ser interpretados como compatíveis com apoio indireto à hipótese de que a persistência de anormalidades perfusionais transitórias, precocemente detectados em pacientes que tiveram ICP bem sucedida no sentido de remover a obstrução coronária em nível subepicárdico, se deva a distúrbios microcirculatórios, pelo menos parcialmente, dependentes de embolização distal. De fato, embora neste estudo a microembolização distal não tenha sido diretamente evidenciada, sabe-se que isso é causado pela aterectomia rotacional, durante o desbaste da placa ocasionada pelo processo ablativo, conforme experimentação direta em animais ${ }^{17,18}$. De forma correlata, estudos conduzidos em humanos demonstraram a ocorrência de anormalidades regionais contráteis e elevação de marcadores de necrose miocárdica, atribuíveis ao processo de microembolização, em pacientes tratados com a aterectomia rotacional ${ }^{19}$.

O processo de microembolização miocárdica decorrente da ICP com aterectomia rotacional, ocasionando os distúrbios perfusionais transitoriamente persistentes na área miocárdica diretamente dependente da artéria coronária tratada, parece envolver tanto o material debridado da placa aterosclerótica, como agregados plaquetários ${ }^{20,21}$. Esta noção tem suporte na constatação de que a inibição da via final comum de agregação plaquetária tenha ocasionado redução significativa da ocorrência dos distúrbios perfusionais verificados durante ICP com aterectomia rotacional ${ }^{22-24}$. Mas, não é possível descartar a ocorrência simultânea de alterações primárias da vasomoção coronária em nível microcirculatório, existentes previamente à ICP, mas persistindo após a remoção do obstáculo hemodinâmico em nível subepicárdico ${ }^{23,24}$.

Os presentes resultados corroboram também o conceito de que, no território vascular a jusante de uma estenose coronária subepicárdica, coexistam anormalidades de regulação de fluxo microvascular ${ }^{25}$. Esses distúrbios, demonstrados com técnicas positrônicas de quantificação do fluxo miocárdico regional, são responsáveis por resposta paradoxalmente vasoconstrictora induzida por taquicardia, em vez da esperada vasodilatação que deveria ocorrer pelo aumento de demanda miocárdica de oxigênio ${ }^{26}$. Outras investigações apóiam também essa noção fisiopatológica, pela mensuração da reserva vasodilatadora coronária, a jusante de estenose coronária subepicárdica, com técnicas baseadas no princípio de Doppler; nestes estudos, mesmo com a correção da estenose por ICP, a reserva vasodilatadora coronária não se normalizava imediatamente, indigitando a persistência temporária de anormalidades microcirculatórias em significante proporção dos pacientes tratados ${ }^{27,28}$. Finalmente, é relevante aduzir que esses distúrbios microvasculares, aqui evocados no contexto de pacientes com reais estenoses coronárias em nível subepicárdica, são correspondentes aos habitualmente encontrados em pacientes com angina e coronárias angiograficamente normais ${ }^{29}$.

À luz dos resultados desta e de outras investigações relatadas na literatura, os distúrbios transitórios da perfusão miocárdica detectados precocemente após ICP podem ser considerados análogos ao fenômeno de "no reflow" observado após recanalização coronária no contexto do infarto agudo do miocárdio ${ }^{30}$; isto é, esses distúrbios perfusionais sinalizam que a despeito de alívio adequado da estenose em nível subepicárdico, isto não se acompanhou de reperfusão miocárdica imediatamente satisfatória, por alterações reológicas e hemodinâmicas microvasculares.

\section{CONCLUSÃO}

Esses resultados são compatíveis com o conceito de que a persistência transitória de defeitos perfusionais após angioplastia coronária bem sucedida seja dependente de distúrbios microcirculatórios associados a microembolização durante o procedimento.

\section{REFERÊNCIAS BIBLIOGRÁFICAS}

1. Gibson RS, Watson DD, Taylor GJ, Crosby IK, Wellons HL, Holt ND, et al. Prospective assessment of regional myocardial perfusion before and after coronary revascularization surgery by quantitative thallium-201 scintigraphy. J Am Coll Cardiol. 1983;1(3):804-15.

2. Reed DC, Beller GA, Nygaard TW, Tedesco C, Watson DD, Burwell LR. The clinical efficacy and scintigraphic evaluation of post-coronary bypass patients undergoing percutaneous transluminal coronary angioplasty for recurrent angina pectoris. Am Heart J. 1989;117(1):60-71.

3. Behrenbeck T, Pellikka PA, Huber KC, Bresnahan JF, Gersh BJ, Gibbons RJ. Primary angioplasty in myocardial infarction: assessment of improved myocardial perfusion with technetium99m isonitrile. J Am Coll Cardiol. 1991;17(2):365-72.

4. Manyari DE, Knudtson M, Kloiber R, Roth D. Sequential thallium-201 myocardial perfusion studies after successful percutaneous transluminal coronary angioplasty: delayed resolution of exercise-induced scintigraphic abnormalities. Circulation. 1988;77(1):86-95.

5. Iskandrian AS, Lemlek J, Ogilby JD, Untereker WJ, Cave V, Heo J. Early thallium imaging after percutaneous transluminal coronary angioplasty: tomographic evaluation during adenosine-induced coronary hyperemia. J Nucl Med. 1992;33(12): 2086-9.

6. Kostkiewicz M, Jarosz W, Tracz W, Przewlocki T, Pieniazek $\mathrm{P}$, Podolec $\mathrm{P}$, et al. Thallium-201 myocardial perfusion imaging in patients before and after successful percutaneous transluminal coronary angioplasty. Int J Cardiol. 1996;53(3): 299-304. 
Marin-Neto JA, et al. Persistência de Distúrbios Perfusionais Miocárdicos após Intervenção Coronária Percutânea com Êxito: Dependência de Fatores Microcirculatórios. Rev Bras Cardiol Invas 2007; 15(2): 107-114.

7. Lima Filho MO, Marin-Neto JA. Condutas relacionadas a intervenções coronárias percutâneas e seguimento clínico ulterior. In: Maciel BC, Marin-Neto JA, eds. Manual de condutas clínicas cardiológicas. 1aㅡ ed. Volume 1. São Paulo:Segmento Farma;2005. p.35-8.

8. Dendale PA, Franken PR, Van Den Heuvel P, Van den Branden F, Bossuyt A. Exercise myocardial perfusion and wall motion imaging to predict recurrence of angina pectoris after successful angioplasty. Acta Cardiol. 1996;51(5):409-23.

9. Kubota S. Serial changes in myocardial perfusion and function after successful percutaneous transluminal coronary angioplasty, examined by stress thallium-201 myocardial scintigraphic and stress radionuclide ventriculographic studies. Kaku Igaku. 1994;31(8):909-19.

10. Watanabe J, Nakamura S, Sugiura T, Takehana K, Hamada $\mathrm{S}$, Miyoshi $\mathrm{H}$, et al. Early identification of impaired myocardial reperfusion with serial assessment of ST segments after percutaneous transluminal coronary angioplasty during acute myocardial infarction. Am J Cardiol. 2001;88(9):956-9.

11. Kamp O, Lepper W, Vanoverschelde JL, Aeschbacher BC, Rovai D, Assayag P, et al. Serial evaluation of perfusion defects in patients with a first acute myocardial infarction referred for primary PTCA using intravenous myocardial contrast echocardiography. Eur Heart J. 2001;22(16):1485-95.

12. Koch KC, Kleinhans E, Klues HG, Schulz G, Sigmund M, Buell $U$, et al. Quantitative assessment of transient regional ischemia during rotational atherectomy. J Nucl Med. 1998;39 (3):402-8.

13. Reiber JHC, Kooijman CJ, Slager CJ. Coronary artery dimensions from cineangiogram: methodology and validation of a computer-assisted analysis procedure. Comp Cardiol. 1984; 131-41.

14. Bertrand ME, Bauteres C, Lablanche JM. Percutaneous coronary rotational angioplasty with the rotablator. In: Topol EJ, ed. Textbook of interventional cardiology. Philadelphia:W.B. Saunders;1994. p.659-67.

15. McFadden EP, Lablanche JM, Bauters C. Rotational atherectomy: an overview. In: Reiber JHC, Serruys P, eds. Progress in quantitative coronary arteriography. Dordrecht, The Netherlands: Kluwer;1994. p.353-66.

16. Benoit T, Vivegnis D, Foulon J, Rigo P. Quantitative evaluation of myocardial single-photon emission tomographic imaging: application to the measurement of perfusion defect size and severity. Eur J Nucl Med. 1996;23(12):1603-12.

17. Hansen DD, Auth DC, Vracko R, Ritchie JL. Rotational atherectomy in atherosclerotic rabbit iliac arteries. Am Heart J. $1988 ; 115(1$ Pt 1):160-5.
18. Hansen DD, Auth DC, Hall M, Ritchie JL. Rotational endarterectomy in normal canine coronary arteries: preliminary report. J Am Coll Cardiol. 1988;11(5):1073-7.

19. Teirstein PS, Warth DC, Hag N, Jenkins NS, McCowan LC, Aubanel-Reidel $\mathrm{P}$, et al. High speed rotational coronary atherectomy for patients with diffuse coronary artery disease. J Am Coll Cardiol. 1991;18(7):1694-701.

20. Bowers TR, Stewart RE, O'Neill WW, Reddy VM, Safian RD. Effect of rotablator atherectomy and adjunctive balloon angioplasty on coronary blood flow. Circulation. 1997;95(5): 1157-64.

21. Farb A, Roberts DK, Pichard AD, Kent KM, Virmani R. Coronary artery morphologic features after coronary rotational atherectomy: insights into mechanisms of lumen enlargement and embolization. Am Heart J. 1995;129(6):1058-67.

22. Influence of a platelet GPIIb/IIla receptor antagonist on myocardial hypoperfusion during rotational atherectomy as assessed by myocardial Tc-99m sestamibi scintigraphy. I Am Coll Cardiol. 1999;33(4):998-1004.

23. Koch KC, Radke PW, Kleinhans E, Ninnemann S, Janssens $U$, Klues HG, et al. Mechanisms of myocardial hypoperfusion during rotational atherectomy of de novo coronary artery lesions and stenosed coronary stents: insights from serial myocardial scintigraphy. J Nucl Cardiol. 2002;9(3):304-11.

24. Sharma SK, Chen V. Coronary interventional devices: balloon, atherectomy, thrombectomy and distal protection devices. Cardiol Clin. 2006;24(2):201-15.

25. Sambuceti G, Marzullo P, Giorgetti A, Neglia D, Marzilli M, Salvadori $\mathrm{P}$, et al. Global alteration in perfusion response to increasing oxygen consumption in patients with single- vessel coronary artery disease. Circulation. 1994;90(4):1696-705.

26. Sambuceti G, Marzilli M, Fedele S, Marini C, L'Abbate A Paradoxical increase in microvascular resistance during tachycardia downstream from a severe stenosis in patients with coronary artery disease: reversal by angioplasty. Circulation. 2001;103(19):2352-60.

27. Wilson RF, Johnson MR, Marcus ML, Aylward PE, Skorton DJ, Collins S, et al. The effect of coronary angioplasty on coronary flow reserve. Circulation. 1988;77(4):873-85.

28. Uren NG, Crake T, Lefroy DC, Silva R, Davies GJ, Maseri A. Delayed recovery of coronary resistive vessel function after coronary angioplasty. J Am Coll Cardiol. 1993;21(3):612-21.

29. Cannon RO $3^{\text {rd }}$, Epstein SE. "Microvascular angina" as a cause of chest pain with angiographically normal coronary arteries. Am J Cardiol. 1988;61(15):1338-43.

30. Rezkalla SH, Kloner RA. No-reflow phenomenon. Circulation. 2002;105(5):656-62. 